
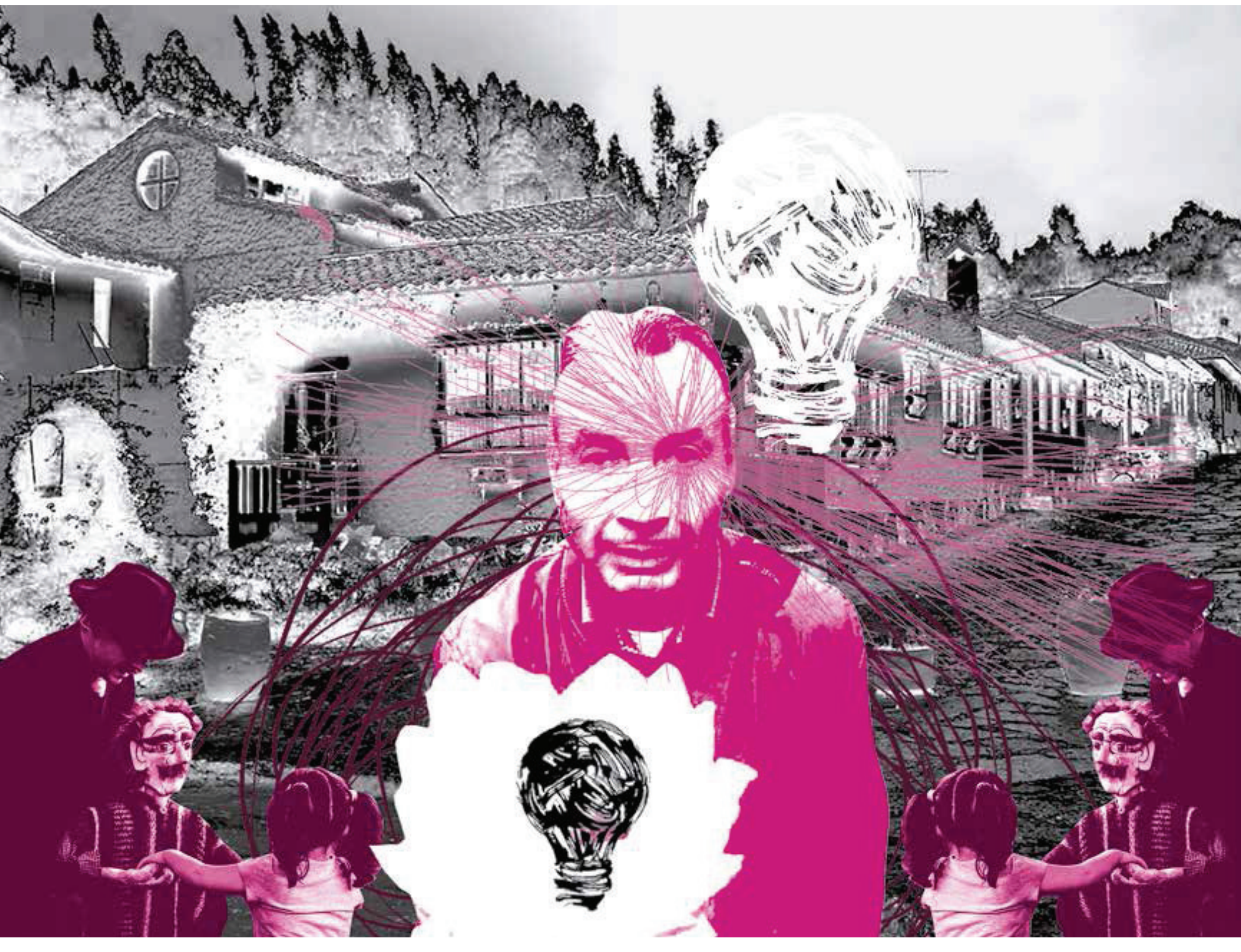

\title{
La innovación de la práctica educativa como lugar de resistencia del maestro
}

The innovation of educational practice as a place of resistance of the teacher Inovação da prática educativa como um lugar de professor de resistência 


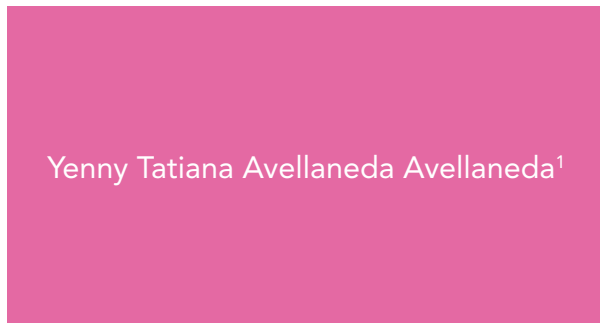

1. Docente Universidad Pedagógica y Tecnológica de Colombia, UPTC; Magíster en Educación, UPTC; correo electrónico: tatiana.av.10@hotmail.com

Fecha de recepción: 24 de febrero de 2017 / fecha de aceptación: 24 de mayo de 2017

\section{Resumen}

Este artículo de investigación presenta elementos de reflexión y análisis sobre los procesos de innovación; es producto de la sistematización de una experiencia desarrollada con comunidades escolares de la provincia de Ricaurte Bajo, departamento de Boyacá, que tuvo como propósito promover, en y desde la escuela, procesos de formación ético política con niños y niñas en contextos de vulneración de derechos ${ }^{2}$.

Palabras clave: Innovación, experiencia, formación política, infancia, escuela.

\section{Summary}

This research article shows a reflection and analysis about innovation processes; it is a product of the systematization of an experience developed with school communities in the province of Ricaurte Bajo, department of Boyacá, whose purpose was to promote, in and from school, processes of ethical political formation with children in contexts of rights' infringement.

Key words: Innovation, experience, political formation, childhood, school.

\section{Resumo}

Este trabalho de pesquisa apresenta elementos de reflexão e análise sobre os processos de inovação; é o produto da sistematização de uma experiência desenvolvida com as comunidades escolares na província de Ricaurte Bajo, departamento de Boyacá, que se destinava a promover, a escola, os processos de formação das crianças ética política em situações de violações de direitos.

Palavras chave: inovação e experiência, formação política, crianças, escola.

2 El trabajo se desarrolló en el marco de la tesis de maestría "La escuela, escenario de formación ético política en contextos de vulneración de derechos", inscrita en la línea de innovaciones pedagógicas de la Maestría en Educación de la Universidad Pedagógica y Tecnológica de Colombia. 


\section{Introducción}

Hoy, cuando el cambio y la transformación se presentan como necesidades imperantes de nuestras sociedades, cada vez más vertiginosas y ávidas de lo novedoso; la innovación debe pensarse más allá del simple hecho del cambiar por cambiar o como oposición a lo tradicional. En tal sentido, uno de los principales desafíos a la hora de hablar sobre innovación, y más aún, de desarrollar una propuesta de innovación, es precisamente definir lo que se puede entender como innovación en el campo educativo.

Para el caso de la experiencia que se presenta, la mirada sobre la innovación toma distancia de la perspectiva técnico-instrumental que la entiende como proceso lineal cuyo propósito es solucionar un problema, haciendo de ella un fin en sí mismo. Por el contrario, se asume una postura crítica que entiende cada innovación como un hecho que se desarrolla en medio de unas condiciones sociales, históricas, culturales e institucionales específicas, por lo cual cuenta en cada caso con una forma particular y propia.

Para la experiencia que se presenta, las condiciones ético políticas de la propuesta de innovación pedagógica "Formación ético política en y desde la escuela", parten del cuestionamiento a la escuela que educa para la competitividad, el éxito y el mundo laboral. En tal sentido, la idea surge en rechazo al modelo de educación que busca educar sujetos que respondan a las pruebas estandarizadas, profundizando así en las desigualdades sociales. En medio de estas condiciones la innovación es entendida como transformación de la escuela desde lo cotidiano; proceso que afecta fundamentalmente la manera en que los sujetos se constituyen a sí mismos en sus interacciones con los otros, dando lugar a la creación de nuevos lugares del ser maestro y del ser niño en un contexto particular.

Esta experiencia se desarrolló con dos comunidades escolares rurales de un municipio de la Provincia de Ricaurte Bajo, departamento de Boyacá; donde la autora trabajó como asesora pedagógica de la Línea de Bienestar Infantil y Juvenil del Programa Ondas-Colciencias Boyacá, acompañando procesos de investigación sobre los derechos de los niños. Con la comunidad se problematizaron y analizaron situaciones, prácticas, visiones y vínculos que tienen lugar al interior de las escuelas, en torno a la relación infancia-escuela-política.

Con el ejercicio se visibilizó una compleja problemática social que se vive a diario en estos contextos, en donde niños y niñas enfrentan diferentes situaciones de vulneración de sus derechos; a partir de ello se reconoce la necesidad de agenciar nuevos procesos educativos, desde un compromiso ético y político con la infancia, en el sentido de la formación de un sujeto que aporte desde su acción y su pensamiento a la transformación de estas condiciones de vida. De esta manera, también se contribuyó al fortalecimiento de la propuesta de la línea de investigación, desde la perspectiva de la formación política.

\section{Construcción de sentido en torno a la innovación}

Hoy en día existen diferentes maneras de entender la innovación, sin embargo, el concepto mismo proviene de los discursos economicistas, bajo la concepción del crecimiento económico irregular (Schumpeter, 1934), el cual considera que para poder mantenerse dentro de un medio competitivo es necesario estar innovando permanentemente. Esta perspectiva ha tenido una gran incidencia en los modelos empresariales hasta nuestros días, sosteniéndose sobre la idea de que la economía capitalista no puede ser ni estacionaria ni uniforme, sino más bien revolucionada por la introducción de nuevas mercancías o formas de producción.

En el contexto latinoamericano, el concepto de innovación aparece en el ámbito educativo hacia la década de los sesenta, asociado a la idea de modernización y entendido como un proceso externo a la escuela, que vive por este tiempo diferentes procesos de reforma. Desde la perspectiva de la modernización escolar, la innovación deviene en la necesidad de formar sujetos productivos para un mundo cada vez más competitivo. Por lo tanto, en adelante se adoptarán políticas de innovación educativa en la región, tendientes a responder a las necesidades impuestas por las nuevas lógicas de la producción de conocimiento y las demandas de la calidad educativa.

En el ámbito educativo el concepto de innovación se asume con diferentes acepciones y sentidos, dependiendo del contexto y la postura que se tenga con respecto a la educación. Su condición de ambiguo, complejo y problemático suscita diferentes formas y discusiones que dependen de la perspectiva desde la cual se le mire. De acuerdo con ello, se pueden encontrar diferentes experiencias cobijadas bajo la concepción de innovación, por lo que no es posible dar una sola definición de la misma, sino reconocer su carácter múltiple y polisémico en cuanto a las formas y sentidos con que es abordado en el campo educativo.

En el caso de la experiencia que se presenta, se entiende la innovación desde la acción y la reflexión sobre la experiencia del maestro, generadoras de nuevos sentidos y significados de la práctica misma; a partir de los cuales se re-contextualizan y construyen nuevos horizontes desde los que se visibilizan y movilizan 
prácticas alternativas que emergen desde los contextos específicos donde se ubica el quehacer del maestro.

En este sentido, y teniendo en cuenta los planteamientos de Mejía (2011), la innovación permite re-contextualizar la práctica y el discurso pedagógico del maestro en el contexto de una práctica social transformadora, desde la cual emergen otras subjetividades y formas de hacer escuela. Para el maestro innovador ello sugiere el reto de redescubrir las posibilidades de su quehacer cotidiano desde nuevas lecturas de la realidad que facilitarán la movilización de nuevas acciones. Así, primero que todo, la innovación se ubica en el sujeto maestro que reconstruye sus concepciones, miradas, prácticas y discursos sobre la realidad educativa, y desde ese cambio de visión irrumpe nuevamente en su realidad con una intención pedagógica transformadora.

En consecuencia, hablamos de la innovación como un proceso abierto e inacabado, construido de manera colectiva, que implica una permanente actitud de búsqueda y la construcción constante de nuevos caminos, es decir, un proceso en continuo movimiento que se activa como respuesta a las realidades sociales que se viven en la escuela, el cual apuesta por la construcción de otras miradas y prácticas sobre lo educativo en confrontación al modelo de educación capitalista.

El cuestionamiento al modelo de educación capitalista y a su interés por fabricar un ciudadano competente, se convierten así en el detonante que permite pensar en otras formas de educar, de construir escuela y de constituirnos como sujetos. Desde esta perspectiva, la innovación se convierte en una manera de hacer resistencia a este tipo de prácticas que promueven la constitución de un sujeto individualizado, autosuficiente, descontextualizado, aislado de sí mismo y del mundo que le rodea; motivado por el éxito y en busca siempre de resultados, que, en el caso del maestro, ha llevado a la mecanización de las prácticas y, en consecuencia, a la imposibilidad de construir un sentido propio sobre lo que hace.

La pregunta se convierte entonces en el punto de quiebre que permite volver a pensar y conectarse con la práctica, reconociéndose como sujeto de voluntad, rompiendo con la continuidad del tiempo medible, calculable, probable, anticipable, para construir el tiempo de la experiencia bajo un nuevo signo, el de la novedad, que trae consigo la posibilidad de renovar-nos en lo que hacemos y hemos sido. En el sentido de lo planteado por Arendt, de inaugurar nuevos comienzos.

Dentro de este marco explicativo será posible construir sentido en torno a la innovación, pensándola como la posibilidad de irrumpir en la cotidianidad escolar con nuevas preguntas, nuevas acciones que permitan construir nuevos significados sobre lo que hacemos como docentes. Innovar la práctica con cada acción en el sentido de hacer posibles nuevos comienzos, viendo la innovación no como un fin en sí mismo, sino como un permanente volver a pensar, a re significar y renovar la propia práctica y, en consecuencia, lo que se ha sido. Se trata de la innovación como la emergencia de algo nuevo posible a través de la acción, la cual se constituye en la actividad política por excelencia.

La acción, pues, en estrecha relación con el discurso, con el poder de la palabra y del lenguaje, es la forma a través de la cual nos insertamos en el mundo, y esa inserción es como un segundo nacimiento cuyo impulso es el comienzo, la capacidad de comenzar, de iniciar, de poner algo en movimiento (Arendt, 2001, p. 68).

Con esta mirada la acción se asume desde un sentido ético-político; en ella se reconoce la capacidad de generar nuevos comienzos desde la facultad de imaginar que las cosas pueden ser de otras maneras a como en realidad son: "El sentido de la política es, entonces, introducir un nuevo comienzo" (Bárcena, 2006, p. 144). La acción también se reconoce como ético-política en cuanto vincula a los sujetos entre sí a partir de su preocupación por un mundo común, en el cual el sujeto asume un lugar desde el cual aporta a su construcción desde un sentido de compromiso y responsabilidad con el otro.

\section{La crisis como condición de posibilidad para la innovación}

"Toda nueva acción implica una cierta destrucción: la eliminación de aquello a partir de lo cual inicia lo que comienza. Ahí residen la fuerza de la acción y su carácter eminentemente revolucionario y político". Bárcena (2006).

La cada vez mayor conciencia sobre el agotamiento en la escuela de las prácticas y discursos escolares mantenidos bajo la lógica del disciplinamiento del cuerpo y la regulación de las conductas, ha permitido a los maestros reconocer los límites de dichos discursos y prácticas a la hora de comprender una realidad, en este caso la educativa; dando lugar a la pregunta como cuestionamiento del orden existente y como posibilidad de pensar otros órdenes. De ahí la fuerza de la cita que da apertura a este apartado, ya que invita a pensar la acción desde una perspectiva política y revolucionaria, en un sentido contestatario y transformador.

En este sentido, se cuestiona el privilegio dado por la escuela a las prácticas de modelamiento de la conducta sobre la formación para la acción, la recitación de los contenidos por encima de la 


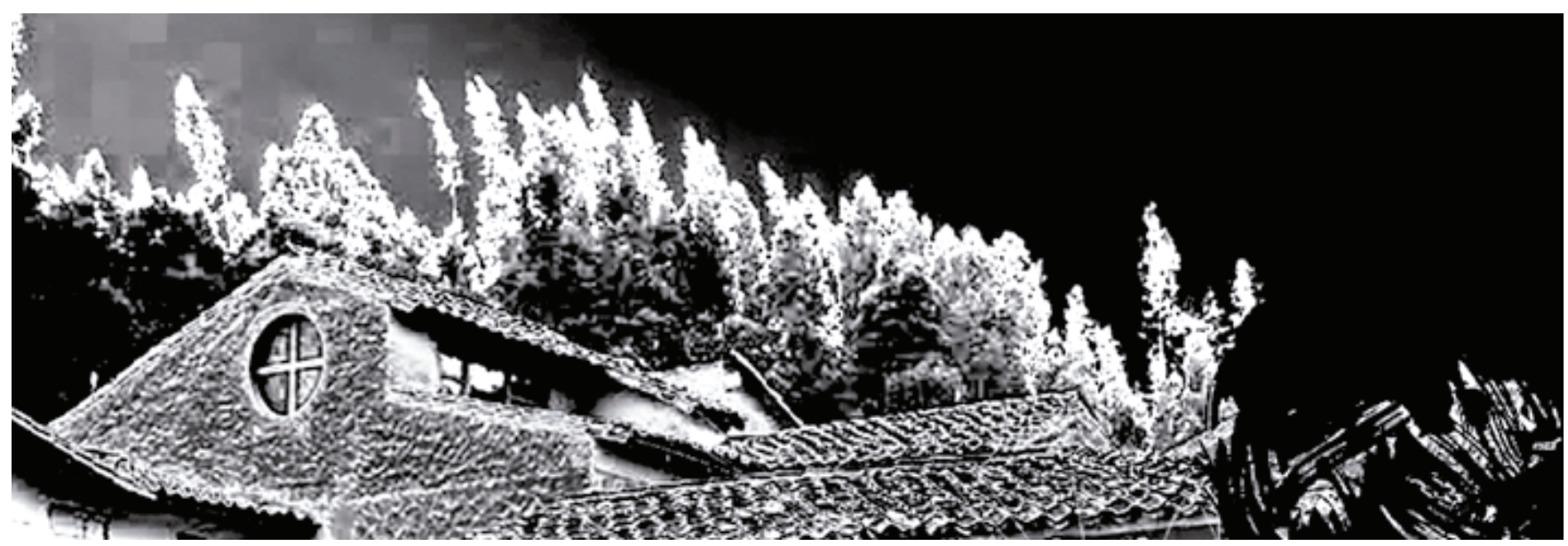

espontaneidad del diálogo y el acontecimiento como novedad, lo racional sobre lo emocional y el individualismo en lugar de la colaboración y la solidaridad.

Cuestionar estas lógicas de poder permite reconocer que mantener estas prácticas de disciplinamiento y regulación no da lugar al desarrollo de procesos de formación que logren afectar las historias de vida de los niños, poniendo en evidencia la actual crisis de sentido que atraviesa la escuela, que no le permite constituirse como referente de actuación para niños y niñas, quienes se ven enfrentados diariamente a dos realidades: el mundo controlado que representa para ellos la escuela y el mundo cada vez más incierto en el que viven. Frente a estas condiciones actuales de la escuela se puede decir que dicha institución, como proyecto de la modernidad, entra en crisis; se cuestiona entonces su función en la sociedad, su carácter disciplinar, su estructura jerárquica y excluyente:

Por ello, este intento innovador/transformador se convierte en el lugar por excelencia del esfuerzo recontextualizador de los conocimientos, discursos y poderes que pugnan por colocarle una acción con sentidos diferentes a los intentos de consolidar una educación y una escuela que le apunten a la construcción de lo social y de lo humano para estos tiempos (Mejía, 2011, p. 43).

Entonces, no es desde la idealización, sino desde la confrontación de la escuela con las realidades sociales, que se ubica el interés por innovar como apuesta política para su construcción como espacio sociocultural de dinamización de propuestas educativas desde referentes éticos, políticos y culturales; en el sentido de lo expresado por Freire (1999), un lugar pertinente de formación de ciudadanos que superen posiciones ingenuas de sí mismos y de su realidad, lo que implica una práctica articulada entre lo que se piensa y la forma como se actúa, ahora caracterizada por dinámicas de diálogo, problematización y trabajo colaborativo, desde los cuales se promuevan transformaciones en el propio sujeto y en "la realidad dada", que ahora es asumida como construcción: Hay otra tarea que cumplir en la escuela a pesar del poder dominante -y por causa de él-, la de desopacar la realidad nublada por la ideología dominante. Obviamente, esta es la tarea de los profesores y profesoras progresistas que saben que tienen el deber de enseñar en forma competente los contenidos, pero que saben también que al hacerlo se obligan a desvelar el mundo de la opresión. Ni contenido solamente, ni desvelamiento solamente -como si fuera posible separarlos-, sino el desvelamiento del mundo opresor por medio de la enseñanza de los contenidos. El cumplimiento de esta tarea progresista implica además la lucha incansable por la escuela pública, por un lado, y por el otro el esfuerzo por ocupar su espacio en el sentido de hacerla mejor. Es esta una lucha que exige claridad política y preparación científica (Freire, 1999, p. 59).

De acuerdo con Freire, la dimensión ético-política de la escuela lleva a que su compromiso y responsabilidad con las comunidades trascienda la enseñanza de contenidos, normas y procedimientos; para constituirse en un referente importante en la vida de los niños y niñas, en la medida en que los procesos de formación despliegan artes de constitución de subjetividad. En Freire se reconoce que una de las funciones relacionadas con la dimensión sociocultural y ética de la escuela está relacionada con 
la formación política, campo desde donde es posible potenciar conocimientos, actitudes y valores, que permitan el desarrollo de los estudiantes como sujetos con posibilidades de pensar, actuar y transformarse a sí mismos y a su realidad cotidiana a través de la participación en sus contextos de actuación.

Para la escuela, esto implica que la formación ético-política debe ir más allá del conocimiento sobre la norma y trascender al ámbito de la formación de subjetividades críticas, en relación con lo cual se asume como insuficiente el solo conocimiento sobre los derechos, en términos de su difusión en el ámbito escolar, ya que se considera necesario un reconocimiento y re significación de los derechos del niño(a) desde su experiencia cotidiana en contextos de vulneración.

Así, la escuela como espacio de formación política permite a estudiantes y maestros construir experiencias que trascienden la lógica disciplinar y de control, con lo cual el docente puede asumirse como tal desde otras miradas que le brinden posibilidades distintas para vincularse con los estudiantes, ya no desde la autoridad y el poder como control, sino desde lenguajes y proyectos compartidos que le permitan ser maestro en el acompañar, mediar, orientar y compartir; encontrando de esta manera un lugar diferente de su oficio, que le permita a su vez pensar su propia práctica, re significarla y finalmente transformarla de acuerdo con las características y necesidades del contexto.

Todo ello supone una problematización de la concepción moderna de infancia, es necesario re significar el vínculo social entre adultos y niños, y movilizar el sentido de lo ético-político en las prácticas educativas a través de la conformación de colectivos escolares con proyectos sociales compartidos y apuestas en común, de tal forma que hagan posible la incidencia de la escuela en las transformaciones sociales de sus comunidades desde el papel de la acción y la palabra. En este sentido, la acción ético-política ejercida, en y desde el escenario escolar, implica una transformación en las relaciones de poder entre niños y adultos, que tradicionalmente ha ejercido el adulto sobre el niño, negándose a reconocer en él su capacidad de pensar y actuar con autonomía y responsabilidad.

Las voces, las historias, formas de pensar, imaginarios y problemáticas de niños y niñas en relación con la experiencia de sus derechos en contextos de vulneración, necesitan tener un lugar visible en las prácticas escolares, ya que no es suficiente el simple conocimiento conceptual e histórico de los derechos para que ellos se sientan y actúen como ciudadanos. Si bien el reconocimiento de los derechos legalmente constituidos es importante en su constitución como sujetos de derechos, la norma no garantiza que se piensen y actúen como tal, menos aún cuando en la cotidianidad de su vida experimentan situaciones de abuso, maltrato, abandono e indiferencia.

Esto hace pensar en la posibilidad de formar al niño(a) en el ejercicio pleno de su ciudadanía apartir de experiencias formativas que le permitan dialogar con el mundo desde un pensamiento y una actitud de reflexión crítica. Permitiéndole ejercer un mayor dominio sobre su propia vida y sus circunstancias, proponiendo acciones creativas en respuesta a sus problemas cotidianos a través de su participación en colectivos infantiles que promuevan experiencias transformadoras para sí mismo y su contexto, mediante la construcción de redes de acción social y política al interior de la vida escolar.

\section{Formación ético-política en y desde la escuela}

Desde la permanente búsqueda de una escuela que movilice procesos de transformación, en el sentido de repensarse e innovar desde sus prácticas educativas, se plantea la propuesta "Formación ético-política en y desde la escuela", centrada en el papel de la acción y la palabra como posibilitadoras de procesos de formación ético-política en niños y niñas desde el desarrollo del pensamiento crítico. La propuesta se diseña y desarrolla a partir de la experiencia del maestro convertida en pregunta, en cuanto cuestiona el ser maestro en contextos de vulneración de derechos, el sentido de la formación en la escuela, la pregunta por la infancia, la educación y la pedagogía.

La idea se fundamenta en el trabajo colectivo que vincula a niños(as) y adultos en experiencias de trabajo colaborativo y dialógico, en el reconocimiento de los contextos que posibilitan su visibilización, y la del maestro, como sujetos situados social e históricamente, y permiten la movilización de prácticas educativas basadas en la participación desde la acción y la palabra.

Con esto mente, la propuesta de innovación plantea el diseño y desarrollo de experiencias formativas desde el reconocimiento y cuestionamiento de la realidad social, llevado a cabo por 
los mismos niños(as), en contextos que presentan problemáticas sociales caracterizadas por la pobreza, la marginación, el maltrato y la violencia; de tal forma que les sea posible desnaturalizar prácticas y discursos comunes relacionados con estos aspectos, para entrar a problematizarlos y luego llegar a proponer y desarrollar acciones conjuntas que faciliten su intervención activa en el cambio de sus condiciones de vida. Así, se busca promover su participación en sus contextos escolares y desarrollar prácticas educativas reflexivas, críticas y transformadoras, a través del reconocimiento de la acción política como hecho que puede afectar la construcción social de realidad desde la acción como novedad.

El proyecto se diseña y desarrolla de manera colectiva, como una propuesta situada, contextualizada y colaborativa que vincula a estudiantes y maestros en torno a la indagación, develamiento y posicionamiento frente a su realidad; permitiéndoles reconocerse y reconocer sus propias problemáticas a partir de procesos de reflexión, problematización y acción que desnaturalicen lugares comunes relacionados con el papel de la escuela en la sociedad, el rol del maestro, la relación entre adultos y niños(as), y el rol de cada uno de estos actores en el contexto escolar.

Se plantea entonces un proceso ético-político de formación, centrado en la acción y la palabra como estrategia pedagógica que potencia en los niños(as) el desarrollo de pensamiento crítico, lo cual implica crear desde la escuela espacios de participación y vinculación en los que se construya, por ellos y para ellos, la posibilidad de relacionarse, hablar, decidir y actuar en sus contextos escolares desde un escenario colectivo y colaborativo, potenciado desde el lenguaje y la acción ético-política creativa que permita resignificar y reconfigurar la vida escolar.

En tal contexto, las preguntas que movilizan el trabajo son: ¿Cómo posibilitar nuevas prácticas educativas que visibilicen las acciones y la palabra de niños y niñas?; ¿a partir de qué acciones se puede movilizar la escuela como contexto de formación ético-política?; ¿cómo puede incidir el maestro(a) desde su práctica educativa en la construcción de procesos de formación de sujetos reflexivos y críticos?; ¿de qué manera pueden incidir los niños y niñas, desde su rol y su saber, en la transformación de sus historias de vida?

De acuerdo con estos interrogantes se proponen tres ciclos de trabajo que funcionan a manera de espiral y que se explicarán a continuación: "Cuestionamiento e indagación desde el pensamiento infantil", "Develamiento de la realidad en torno a la vulneración de derechos" y "Posicionamiento o reconocimiento de posibilidades de acción”.

\section{Cuestionamiento e indagación desde el pensamiento infantil}

Se desarrolló a partir del permanente proceso de preguntar, preguntarse, acerca de sí mismo y del mundo; para ello se recurrió a interrogantes formulados por los niños(as), en los cuales, con mucha curiosidad, fueron aflorando el asombro y el interés por situaciones de su vida cotidiana. Un permanente ejercicio que empleó la pregunta como detonante del pensamiento crítico, permitió desarrollar el siguiente proceso:

Figura 1. Proceso de indagación

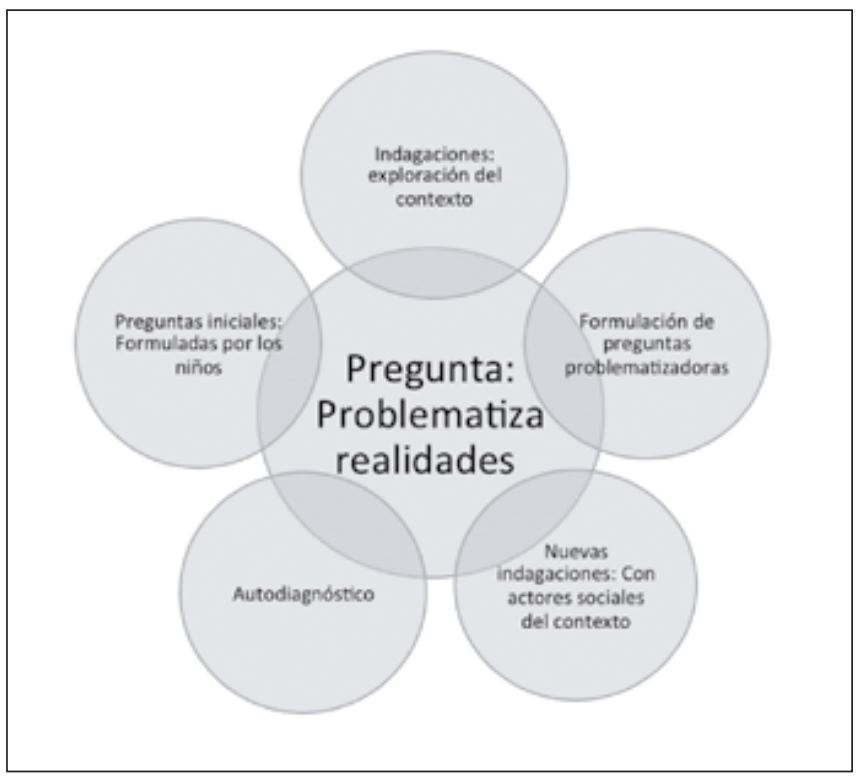

Fuente: Elaborado por el autor

Aunque inicialmente no fue sencillo lograr que los niños manifestaran sus intereses y curiosidades a través de preguntas, debido a su poca familiaridad con esta práctica, poco a poco fueron pasando de afirmaciones como "los niños son maltratados por los adultos", "la escuela es aburrida" o "los compañeros no comparten", a cuestionamientos como "¿Por qué algunos padres abandonan a sus hijos?", "¿qué podemos hacer los niños para que en la escuela pasen cosas divertidas?”, “¿qué actividades nos llevan a no ser envidiosos?”. En este sentido, se considera que las preguntas posibilitan la movilización de los saberes de niños y niñas, hacia la búsqueda de nuevos lugares que superen visiones ingenuas de la realidad. 


\section{Develamiento de la realidad en torno a la vulneración de derechos}

A través de espacios de discusión colectiva se promovieron procesos de problematización y reflexión sobre las propias historias de los niños(as). Estas discusiones les permitieron identificar y cuestionar situaciones de conflicto, abandono e indiferencia en sus contextos escolares y familiares, problematizando así las formas de poder, de control, de imposición y sometimiento en las relaciones entre adultos y niños, naturalizadas en las prácticas cotidianas. Este desciframiento de la realidad a partir de las preguntas y problemas construidos por alumnos y maestros, plantea procesos de emancipación desde la posibilidad de cuestionar y transformar el orden impuesto, desde nuevas miradas de la realidad. El ejercicio se desarrolló a través de las siguientes acciones y ejes pedagógicos:

Figura 2. Proceso de descifrado de la realidad

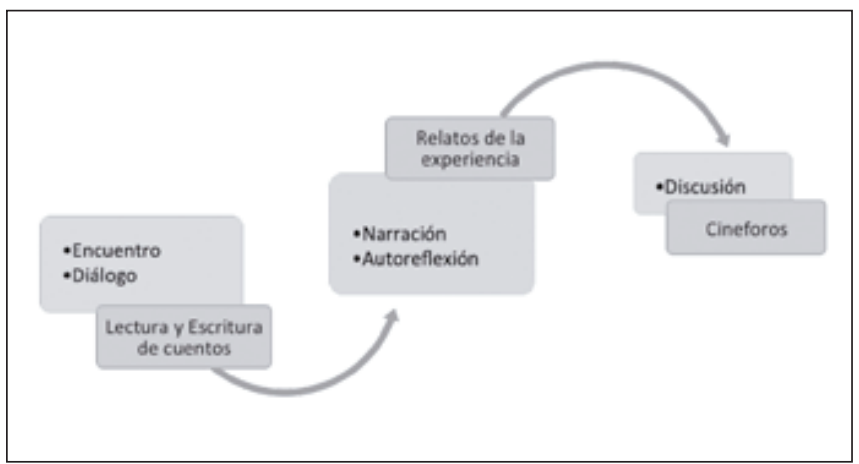

Fuente: Elaborado por el autor

\section{Posicionamiento o reconocimiento de posibilidades de acción}

A partir de los procesos de reflexión y análisis desarrollados previamente por los niños(as), comienzan a reconocer sus propias posibilidades de acción frente a las problemáticas presentadas en los contextos escolar y familiar, movilizándolas a través de prácticas transformadoras de sentidos y significados. De esta manera, la elaboración y discusión de mapas sociales, desde la perspectiva de la cartografía social, permitió reconstruir y recrear las problemáticas presentadas en el contexto, visibilizando situaciones, imaginarios, roles y vínculos que hacen parte de la cotidianidad escolar; abriendo así un espacio importante para la comunicación, la participación y el reconocimiento del lugar que cada uno ocupa en el contexto como actor social.

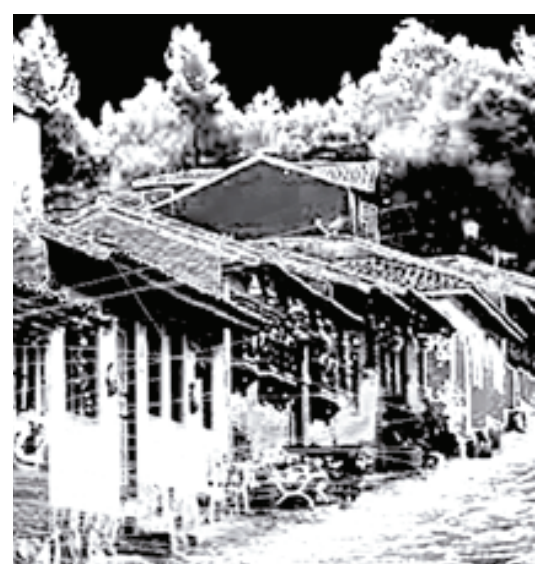

Figura 3. Proceso de reconocimiento de posibilidades de acción

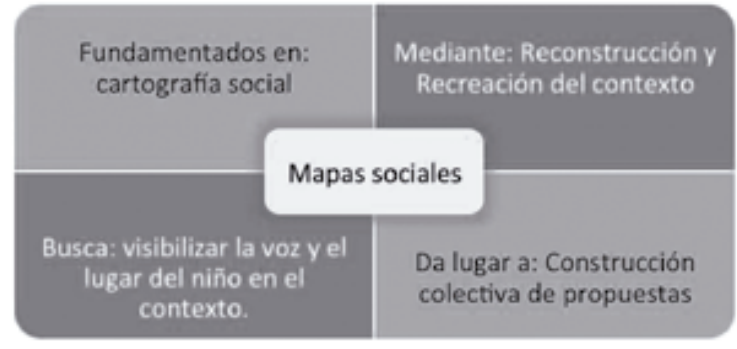

Fuente: Elaborado por el autor

De esta manera fue posible reconocer las posibilidades de acción de niños(as) y maestros frente a las problemáticas del contexto escolar, plasmadas mediante el diseño de rutas de acción y movilizadas a través de prácticas educativas transformadoras de sentidos y significados de lo cotidiano de la vida escolar. Así, se reconoce que en los niños(as) la acción política es creativa y que con ella se rompe con la rutina escolar, instaurando nuevas formas de vinculación entre el sujeto y su contexto. Es una acción innovadora, en el sentido que posibilita nuevos comienzos, es inventiva, construye otros caminos. 
- educación " 32

\section{Referencias}

Arendt, H. (2001). ¿Qué es la política? Barcelona: Paidós.

Arendt, H. (2009). La condición humana. Barcelona: Paidós.

Bárcena, F. (2006). Hannah Arendt: una filosofía de la natalidad. Barcelona: Herder.

Freire, P. (1999). Política y educación. México: Siglo Veintiuno Editores.

Mejía, M. R. (2011). La(s) escuela(s) de la(s) globalización(es) II. Entre el uso técnico instrumental y las educomunicaciones. Bogotá: Ediciones Desde Abajo. 